\title{
Network pharmacology-based prediction of the active compounds and mechanism of Buyang Huanwu Decoction for ischemic stroke
}

\author{
KAI WANG ${ }^{1,2^{*}}$, LU LEI $^{1 *}$, JINYI CAO $^{1 *}$, YI QIAO $^{1,3^{*}}$, RUIMIN LIANG $^{2}$, JIALIN DUAN $^{1}$, \\ ZHIJUN FENG ${ }^{1}$, YI DING ${ }^{1}$, YANG MA ${ }^{1}$, ZHIFU YANG ${ }^{1}$ and ENHU ZHANG ${ }^{2}$ \\ ${ }^{1}$ Department of Pharmacy, Xijing Hospital, Fourth Military Medical University, Xi'an, Shaanxi 710032; \\ ${ }^{2}$ College of Pharmacy, Shaanxi University of Chinese Medicine, Xianyang, Shaanxi 712046; \\ ${ }^{3}$ Department of Pharmacology, Xi'an Jiaotong University School of Medicine, Xi'an, Shaanxi 710061, P.R. China
}

Received June 19, 2020; Accepted February 9, 2021

DOI: $10.3892 / \mathrm{etm} .2021 .10484$

\begin{abstract}
Buyang Huanwu Decoction (BYHWD) is used to promote blood circulation and is widely used in Chinese clinical practice for the treatment and prevention of ischemic cerebral vascular diseases. However, the mechanism and active compounds of BYHWD used to treat ischemic stroke are not well understood. The current study aimed to identify the potential active components of BYHWD and explore its mechanism using network pharmacology and bioinformatics analyses. The compounds of BYHWD were obtained from public databases. Oral bioavailability and drug-likeness were screened using the absorption, distribution, metabolism and excretion (ADME) criteria. Components of BYHWD, alongside the candidate targets of each component and the known therapeutic targets
\end{abstract}

Correspondence to: Professor Zhifu Yang, Department of Pharmacy, Xijing Hospital, Fourth Military Medical University, 127 Changle West Road, Xincheng, Xi'an, Shaanxi 710032, P.R. China

E-mail: yangtian_1973@163.com

Professor Enhu Zhang, College of Pharmacy, Shaanxi University of Chinese Medicine, 1 Middle Section, Chenyangzhai Century Avenue, Qindu, Xianyang, Shaanxi 712046, P.R. China

E-mail: 824887855@qq.com

${ }^{*}$ Contributed equally

Abbreviations: BYHWD, Buyang Huanwu Decoction; ADME, absorption, distribution, metabolism, and excretion; TCM, traditional Chinese medicine; OB, oral bioavailability; DL, drug-likeness; KEGG, Kyoto Encyclopedia of Genes and Genomes; GO, Gene Ontology; PPI, protein-protein interaction; BMECs, brain microvascular endothelial cells; OGD, oxygen glucose deprivation; $\mathrm{BP}$, biological process; MF, molecular function; $\mathrm{CC}$, cellular component; HIF-1 $\alpha$, hypoxia-inducible factor 1-alpha; TLRS, toll-like receptors

Key words: traditional Chinese medicine, Buyang Huanwu decoction, network pharmacology, cerebral ischemia, pharmacological mechanism of ischemic stroke were collected. A network of target gene compounds and cerebral ischemia compounds was established using network pharmacology data sources. The enrichment of key targets and pathways was analyzed using STRING and DAVID databases. Moreover, three of key targets [IL6, VEGFA and hypoxia-inducible-factor-1 $\alpha$ (HIF-1 $\alpha$ )] were verified using western blot analysis. Network analysis determined 102 compounds in seven herbal medicines that were subjected to ADME screening. A total of 42 compounds as well as 79 genes formed the principal pathways associated with ischemic stroke. The 16 key compounds identified were baicalein, beta-carotene, baicalin, kaempferol, luteolin, quercetin, hydroxysafflor yellow A, isorhamnetin, bifendate, formononetin, calycosin, astragaloside IV, stigmasterol, sitosterol, Z-ligustilide, and dihydrocapsaicin. The core genes in this network were IL6, TNF, VEGFA, HIF-1 $\alpha$, MAPK1, MAPK3, JUN, STAT3, IL1B and IL10. Furthermore, the TNF, IL17, apoptosis, PI3K-Akt, toll-like receptor, MAPK, NF-кB and HIF-1 signaling pathways were identified to be associated with ischemic stroke. Compared with the control group (no treatment), BYHWD significantly inhibited the expression of IL6 and increase the expression of HIF-1 $\alpha$ and VEGFA. Network pharmacology analyses can help to reveal close interactions between multi-components and multi-targets and enhance understanding of the potential effects of BYHWD on ischemic stroke.

\section{Introduction}

Ischemic stroke, also known as cerebral ischemia, is an acute cerebrovascular disease that is associated with high disability and mortality rates $(1,2)$. According to statistics compiled by the American Heart Association in collaboration with the National Institutes of Health and other government agencies, stroke has become the third most common cause of death worldwide after cancer and heart disease in recent years, placing a huge psychological and financial burden on many families (3).

Buyang Huanwu decoction (BYHWD) has been used to promote blood circulation and was proposed by Wang Qingren in the 'Correction on Errors in Medical Classic'. It is composed of Radix Astragali, the dried roots of 
Astragalus membranaceus Bge; Angelicae Sinensis Radix, the dried roots of Angelica sinensis Diels; Paeoniae Radix Rubra, the dried roots of Paeonia lactiflora Pall; Chuanxiong Rhizoma, the dried rhizomes of Ligusticum chuanxiong Hort; Persicae Semen, the dried seeds of Prunus persica Batsch; Carthami Flos, the dried flowers of Carthamus tinctorius L; and Pheretima, the dried bodies of Pheretima aspergillum (E.Perrier), in the ratio of 120:6:4.5:3:3:3:3. This formula has been widely used in Chinese clinical practice for the treatment and prevention of ischemic cardio-cerebral vascular diseases (4). Previous studies have demonstrated that BYHWD is efficacious in treating ischemic stroke and other vascular diseases (5). However, the exact mechanism of BYHWD in improving ischemic stroke and its active components remain unclear. As such, there is a great interest in identifying the active compounds and molecular targets in BYHWD that act on ischemic stroke. The present study aimed to identify the potential active constituents of BYHWD and explore its mechanism of action in the amelioration of ischemic stroke.

BYHWD is a multi-component, multi-channel and multi-target agent that is a product under the guidance of the holistic view and dialectical theory (6). It has unique advantages for the treatment of complex diseases, but due to the complex composition of TCM compounds, the modernization process can be slow (7). Network pharmacology is a novel method that combines system network analysis and pharmacology that can be used to clarify the synergistic effects and underlying mechanisms of compound-compound, compound-target and target-disease networks at the molecular level. This helps to elucidate the interactions among compounds, genes, proteins and diseases $(8,9)$. Due to its robust and systematic nature, which is consistent with the overall view of TCM and the principle of dialectical theory, it has been widely applied in TCM research (10). Therefore, the current study used network pharmacology to study the mechanism of BYHWD in the treatment of ischemic stroke, providing a reference and theoretical basis for its experimental research and clinical application.

\section{Materials and methods}

Chemical database collection of BYHWD-compound information. Information on the compounds included in BYHWD was collected from two phytochemical databases, the TCM systems pharmacology database (TCMSP, http://ibts.hkbu. edu.hk/LSP/tcmsp.php) and the TCM Database@Taiwan (http://tcm.cmu.edu.tw/). The bidimensional chemical structures were acquired from NCBI PubChem (http://pubchem. ncbi.nlm.nih.gov/).

Pharmacokinetic ADME evaluation. OB represents the ability of a compound to circulate in the body after oral administration. OB can indicate whether the active compounds in a formula can be delivered throughout the body and produce a physicochemical effect (11). DL is an indicator for determining the similarity or likeness of a compound and its physicochemical properties with conventional drugs. DL can help determine if a certain compound has a therapeutic effect (11). All compounds were selected using the in silico integrative ADME model provided by the TCMSP database. Chemicals without ADME information were removed from the final list.
Compounds were only retained if $\mathrm{OB} \geq 30$ and $\mathrm{DL} \geq 0.18$ to satisfy the criteria suggested by the TCMSP database (12).

Target genes related to the identified compounds. The TCMSP database is a unique pharmacological database of traditional Chinese medicines describing the relationships between drugs, targets and diseases. The name of the identified compound was input into the TCMSP database version 2.3 (https://tcmspw.com/tcmsp.php) to obtain its target name, after which the target name of each compound was input into the UniProt (http://www.uniprot.org/) database to convert to the target gene and confine the species to Homo sapiens. Subsequently, the various IDs of the targets were transformed into UniProt IDs (13).

Collection of the cerebral ischemia target genes. Therapeutic Target Database (TTD) (https://db.idrblab.org/ttd/) explores therapeutic protein and nucleic acid targets within a targeted disease (14). DisGeNET (http://www.disgenet.org/web/ DisGeNET/menu/home) is a discovery platform containing one of the largest publicly available collections of genes and variants associated with human diseases (15). By searching the key word 'cerebral ischemia', the targets related to this disease were collected from each database.

Construction of the networks and pathway analyses. The constructed herbal-chemical-protein networks were visualized using Cytoscape version 3.6.1 (http://www.cytoscape.org/). The relationship between active ingredients and common targets of component diseases in BYHED were summarized into a table and imported into Cytoscape software to obtain a network of drug ingredient actions and disease targets. The nodes in the networks represent herbs and chemicals, and the edges indicated interactions between herbs and chemicals, and between chemicals and target genes (16). The common targets of components and diseases were imported into the ClueGo plug-in for Cytoscape software. The main functional annotation clusters were ranked using the Biocarta functional annotation cluster tool (http://amp.pharm.mssm. edu/Harmonizome/dataset/Biocarta+Pathways; updated on July 3, 2016) (17). The functional pathways of BYHWD associated with cerebral ischemia were analyzed using Kyoto Encyclopedia of Genes and Genomes (KEGG) pathway analysis (http://www.genome.jp/kegg/pathway.html). Gene Ontology (GO) enrichment evaluation was determined using the DAVID database version 6.8 (https://david.ncifcrf.gov/). Data with $\mathrm{P}<0.05$ were screened from the enrichment analysis results.

Protein-protein interaction (PPI) analysis. Screened drug component-disease common targets were imported into the STRING (https://string-db.org/) database to build the PPI network model. The protein category was set as Homo sapiens. The minimum interaction threshold was set as the medium highest confidence $(>0.9)$ and the PPI network was obtained using the default setting of the other parameters. In the network, the size of the nodes represented the degree size. The higher the degree, the better the correlation between the protein and therapeutic mechanism (18). Fig. 1 illustrated network pharmacology analysis workflow. 


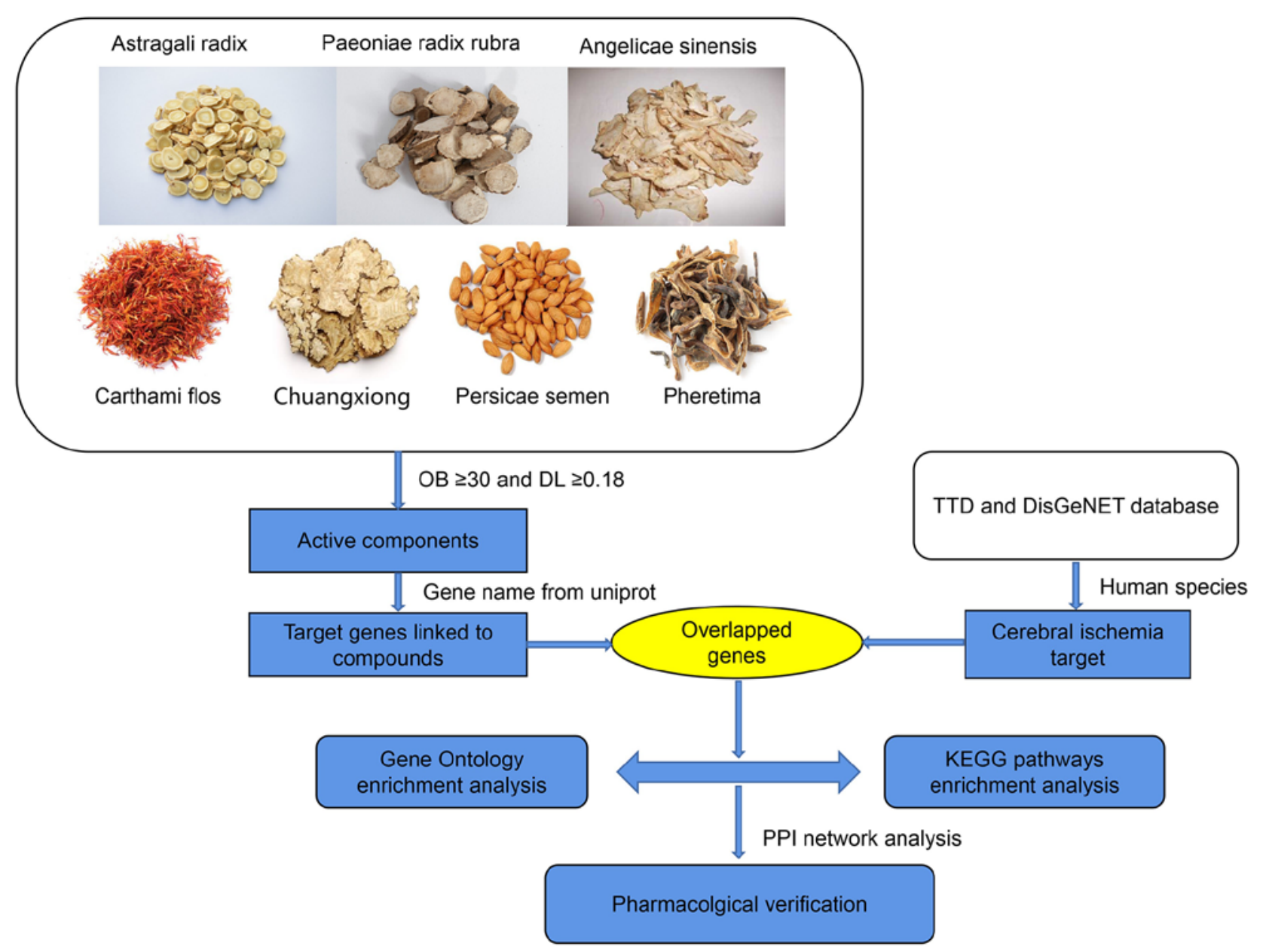

Figure 1. Workflow of network pharmacology analysis. OB, oral bioavailability; DL, drug-likeness; TTD, Therapeutic Target Database; KEGG, Kyoto Encyclopedia of Genes and Genomes; PPI, protein-protein interaction.

Materials for pharmacological experimental verification. BYHWD is a granule made of Astragali Radix, Angelicae Sinensis Radix, Paeoniae Radix Rubra, Chuanxiong Rhizoma, Persicae Semen, Carthami Flos and Pheretima according to the ratio 120:6:4.5:3:3:3:3. BYHWD was obtained from Guangdong Yifang Pharmaceutical Co., Ltd._batch no. 7070352). Rat brain microvascular endothelial cells (BMECs) were purchased from the Cell Biologics, Inc. (cat. no. C57-6023). Primary antibodies against $\beta$-actin (cat. no. ab227387), VEGFA (cat. no. ab46154), hypoxia-inducible-factor-1 $\alpha$ (HIF-1 $\alpha$ ) (cat. no. ab179483) and IL6 (cat. no. ab9324) and secondary antibodies [HRP-conjugated goat anti-rabbit (cat. no. ab7090) and HRP-conjugated goat anti-mouse (cat. no. ab97040)] were purchased from Abcam.

Cell culture and treatments. BMECs were divided into three groups: Control, oxygen-glucose deprivation (OGD) and OGD group treated with BYHWD $(80 \mu \mathrm{g} / \mathrm{ml})$ groups. OGD was established as follows: Cells were rinsed once with glucose-free DMEM (Gibco; Thermo Fisher Scientific, Inc.) (19) and transferred to an anaerobic chamber (Thermo Fisher Scientific, Inc.) containing a gas mixture composed of $7 \% \mathrm{CO}_{2}$ and $93 \% \mathrm{~N}_{2}$ for $6 \mathrm{~h}$ at $37^{\circ} \mathrm{C}$. The cells were then returned to the normal culture conditions $\left(37^{\circ} \mathrm{C}\right)$. Control BMECs were cultured in complete DMEM under normal conditions $\left(37^{\circ} \mathrm{C}\right)$ and did not receive
BYHWD treatment. BYHWD was dissolved in complete DMEM and treated with a $0.22 \mu \mathrm{m}$ membrane filter, after which BYHWD group cells were treated $\left(37^{\circ} \mathrm{C}\right)$ with $80 \mu \mathrm{g} / \mathrm{ml}$ BYHWD for $12 \mathrm{~h}$.

Western blotting. BMECs were lysed with cold radioimmunoprecipitation assay buffer (Sigma-Aldrich; Merck KGaA) for $30 \mathrm{~min}$ and protein concentrations were analyzed by the BCA method. Whole-cell lysates $(30 \mu \mathrm{g})$ were fractionated by $10 \%$ SDS-PAGE and transferred onto polyvinylidene fluoride membranes (EMD Millipore). The membranes were blocked with $5 \%$ bovine serum albumin (Beijing Solarbio Science \& Technology Co., Ltd.) at room temperature for $30 \mathrm{~min}$ and incubated overnight at $4^{\circ} \mathrm{C}$ with primary antibodies against VEGFA (1:1,000; anti-rabbit), HIF-1 $\alpha$ (1:1,000; anti-rabbit), IL6 (1:1,000; anti-mouse), $\beta$-actin (1:1,000; anti-rabbit). The membranes were subsequently incubated with a secondary antibody (1:10,000; goat anti-rabbit or goat anti-mouse antibody) at $37^{\circ} \mathrm{C}$ for $1 \mathrm{~h}$. The blots were visualized using an ECL-Plus reagent (Santa Cruz Biotechnology, Inc.) and analyzed using Quantity One System image analysis software version 4.6.2 (Bio-Rad Laboratories, Inc.).

Statistical analysis. All data in the present study were expressed as the mean \pm SD. One-way ANOVA followed by 
a Tukey's post hoc test was used for analyzing differences between groups. $\mathrm{P}<0.05$ was indicated as the statistical significance.

\section{Results}

Compounds in the herbal medicines. BYHWD consists of seven herbal medicines, namely Astragali Radix, Angelicae Sinensis Radix, Paeoniae Radix Rubra, Chuanxiong Rhizoma, Persicae Semen, Carthami Flos and Pheretima. All identified compounds were subjected to ADME screening. Of the 780 compounds assessed, 102 had an $\mathrm{OB} \geq 30 \%$ and $\mathrm{DL} \geq 0.18$, which met the suggested drug screening criteria. Of these, 21, 23, 4, 29, 9, 23 and 5 were Astragali Radix, Carthami Flos, Angelicae Sinensis Radix, Paeoniae Radix Rubra, Chuanxiong Rhizoma, Persicae Semen and Pheretima, respectively. There were 6 compounds that did not meet the aforementioned requirements and were therefore considered to be bioactive compounds: Hydroxysafflor yellow A, astragaloside IV, ferulic acid, ligustrazine, Z-ligustilide and linoleic acid. These compounds are the major components of BYHWD and their effects on cerebral ischemia have been investigated previously (20-25). Duplicate components and those with ambiguous targets were removed. The compound information of BYHWD is presented in Table I, and the composition of each structure is presented in Fig. 2.

Combination of the compound-target and cerebral ischemia target genes. A total of 507 genes and compounds related to BYHWD were obtained from the TCMSP database (Table SI). A total of 274 genes associated with cerebral ischemia were retrieved from the TTD and DisGeNET version 6.0 databases (Table SII). A total of 79 overlapping genes were pinpointed by matching the 518 compound genes with the disease-associated genes (Fig. 3). Therefore, these target genes were used as prediction targets of BYHWD in the treatment of stroke.

Potential target genes and network analysis. The constructed herbal-chemical-protein networks were visualized using Cytoscape, which included 42 compounds, with 79 target genes, 129 nodes and 490 edges (Fig. 4). In particular, 16 of the compounds (baicalein, beta-carotene, baicalin, kaempferol, luteolin, quercetin, hydroxysafflor yellow A, isorhamnetin, bifendate, formononetin, calycosin, astragaloside IV, stigmasterol, sitosterol, Z-ligustilide and dihydrocapsaicin) were associated with $>5$ genes. Additionally, 33 genes (PTGS2, MMP9, NOS2, MAPK1, MAPK3, TNF, EGFR, APP, JUN, TP53, IL6, IL2, STAT3, NOS3, IL10, CYCS, VEGFA, HIF-1 $\alpha$, BCL2, CASP9, CAT, PARP1, CASP3, CCL2, MPO, NFE2L2, CSF2, RELA, ALB, NFKBIA, PPARG, NR3C2 and ICAM1) were regulated by $>4$ compounds. The compound-target gene network demonstrated intimate communication between several components and multiple targets. This is helpful to better understand the potential pharmacodynamic substances and targets of BYHWD in the treatment of cerebral ischemia.

GO and KEGG enrichment analysis of potential target genes. GO enrichment and KEGG pathway enrichment analyses of the 79 potential targets were performed to determine the underlying molecular mechanism of BYHWD in cerebral ischemia.
GO enrichment analysis provided biological process (BP), molecular function (MF) and cellular component (CC) results. Using the DAVID database (with $\mathrm{P}<0.05$ ), the results revealed that the main BPs were 'positive regulation of transcription from RNA polymerase II promoter', 'inflammatory response', 'negative regulation of apoptosis', 'angiogenesis', 'positive regulation of NF-kB transcription factor activity', 'apoptosis process', 'immune response' and 'response to oxidative stress'. The MFs were mainly associated with 'DNA binding', 'transcription factor activity', 'heparin-binding', 'heme-binding', sequence-specific DNA binding' and 'identical protein binding'. CC analysis revealed that there was a higher proportion of protein in the nucleus and extracellular space. Furthermore, GO analysis revealed the top six enriched conditions in the BP, CC and MF categories (Fig. 5). Furthermore, the 20 BPs are listed in Table II. To examine the signaling pathways and functions of these target genes, KEGG pathway functional enrichment analysis was performed. The signaling pathways were obtained by screening their statistical significance $(\mathrm{P}<0.05)$, and the resulting target genes were found to primarily interact with the TNF, IL17, PI3K-Akt, toll-like receptor (TLR), MAPK, NF- $\kappa \mathrm{B}$ and HIF-1 signaling pathways. Therefore, these signaling pathways appear to be closely associated with the potential effects of BYHWD in cerebral ischemia. The identified target genes are listed in Table III. A KEGG bubble diagram was created using the top 20 signals (Fig. 6A). Additionally, the main functional annotation clusters were ranked by the Biocarta functional annotation cluster tool and were presented in Fig. 6B.

PPI network analysis of the target genes. The drug component-disease common target was imported into the STRING database to construct the PPI network. When using an interaction score of $>0.9$ as a cut-off, the PPI network contained 79 nodes and 327 edges, with an average node degree of 8.28 and PPI enrichment P-value of $<1.0 \times 10^{-16}$ (Fig. 7A). Cytoscape version 3.6.1 was then used to analyze the degree, PPI network topological eigenvalues, degree of node color, size reaction center, edge thickness and color depth to determine a combined score. The top 20 target genes with a high degree of connectivity were selected as the hub genes of BYHWD for cerebral ischemia (Fig. 7B). The core genes in this network were IL6, TNF, VEGFA, HIF-1 $\alpha$, MAPK1, MAPK3, JUN, STAT3, IL1B and IL10.

Experimental validation. To confirm the results of the network analysis and verify the key targets of BYHWD, three key targets (IL6, VEGFA and HIF-1 $\alpha$ ) were selected for pharmacological validation (Fig. 8). Western blotting results revealed that, when compared with the control group, BYHWD significantly inhibited the expression of IL6 and increased the expression of HIF-1 $\alpha$ and VEGFA $(\mathrm{P}<0.05)$.

\section{Discussion}

Ischemic stroke is one of the leading causes of death and disability worldwide. Previous studies have indicated that the injury mechanism of ischemic stroke includes apoptosis, necrosis, inflammation, immune regulation and oxidative stress (26). However, no effective treatment has been found 
Table I. Final selected compounds among the seven herbal medicines of Buyang Huanwu Decoction.

\begin{tabular}{|c|c|c|c|c|}
\hline No. & Compound & OB $(\%)$ & DL & Herbal medicine \\
\hline 1 & Kaempferol & 41.88 & 0.24 & Astragali Radix, Carthami Flos \\
\hline 2 & Quercetin & 46.43 & 0.28 & Astragali Radix \\
\hline 3 & Mairin & 55.38 & 0.78 & Astragali Radix \\
\hline 4 & Jaranol & 50.83 & 0.29 & Astragali Radix \\
\hline 5 & Hederagenin & 36.91 & 0.75 & Astragali Radix, Persicae Semen \\
\hline 6 & Isorhamnetin & 49.60 & 0.31 & Astragali Radix \\
\hline 7 & Bifendate & 31.10 & 0.67 & Astragali Radix \\
\hline 8 & Formononetin & 69.67 & 0.21 & Astragali Radix \\
\hline 9 & Isoflavanone & 109.99 & 0.30 & Astragali Radix \\
\hline 10 & Calycosin & 47.75 & 0.24 & Astragali Radix \\
\hline 11 & AstragalosideIV & 17.74 & 0.15 & Astragali Radix, Persicae Semen \\
\hline 12 & Poriferast-5-en-3beta-ol & 36.91 & 0.75 & Carthami Flos \\
\hline 13 & Lignan & 43.32 & 0.65 & Carthami Flos \\
\hline 14 & Phytoene & 39.56 & 0.50 & Carthami Flos \\
\hline 15 & Phytofluene & 43.18 & 0.50 & Carthami Flos \\
\hline 16 & Pyrethrin II & 48.36 & 0.35 & Carthami Flos \\
\hline 17 & 6-Hydroxykaempferol & 62.13 & 0.27 & Carthami Flos \\
\hline 18 & Baicalein & 33.52 & 0.21 & Carthami Flos, Paeoniae Radix Rubra \\
\hline 19 & Quercetagetin & 45.01 & 0.31 & Carthami Flos, Paeoniae Radix Rubra \\
\hline 20 & Beta-carotene & 37.18 & 0.58 & Carthami Flos \\
\hline 21 & Baicalin & 40.12 & 0.75 & Carthami Flos,Paeoniae Radix Rubra \\
\hline 22 & Beta-sitosterol & 36.91 & 0.75 & $\begin{array}{l}\text { Carthami Flos,Paeoniae Radix Rubra, } \\
\text { Angelicae sinensis Radix }\end{array}$ \\
\hline 23 & Stigmasterol & 43.83 & 0.76 & $\begin{array}{l}\text { Carthami Flos, Paeoniae Radix Rubra, } \\
\text { Angelicae sinensis Radix }\end{array}$ \\
\hline 24 & Luteolin & 36.16 & 0.25 & Carthami Flos \\
\hline 25 & Ferulic acid & 39.56 & 0.06 & Angelicae sinensis Radix \\
\hline 26 & Cis-ligustilide & 36.91 & 0.75 & Angelicae sinensis Radix \\
\hline 27 & Ellagic acid & 43.06 & 0.43 & Paeoniae Radix Rubra \\
\hline 28 & Paeoniflorin & 53.87 & 13.88 & Paeoniae Radix Rubra \\
\hline 29 & Sitosterol & 36.91 & 0.75 & $\begin{array}{l}\text { Paeoniae Radix Rubra, Chuangxiong } \\
\text { Rhizoma }\end{array}$ \\
\hline 30 & Spinasterol & 42.98 & 0.76 & Paeoniae Radix Rubra \\
\hline 31 & $(+)$-catechin & 54.83 & 0.24 & Paeoniae Radix Rubra \\
\hline 32 & Stigmast-7-en-3-ol & 37.42 & 0.75 & Paeoniae Radix Rubra \\
\hline 33 & Ethyl oleate (NF) & 32.40 & 0.19 & Paeoniae Radix Rubra \\
\hline 34 & Campest-5-en-3beta-ol & 37.58 & 0.71 & Paeoniae Radix Rubra \\
\hline 35 & Hydroxysafflor yellow A & 4.77 & 0.68 & Carthami Flos \\
\hline 36 & Perlolyrine & 65.95 & 0.27 & Chuangxiong Rhizoma \\
\hline 37 & Wallichilide & 42.31 & 0.71 & Chuangxiong Rhizoma \\
\hline 38 & Ligustrazine & 20.01 & 0.03 & Chuangxiong Rhizoma \\
\hline 39 & Z-ligustilide & 53.72 & 0.07 & Chuangxiong Rhizoma \\
\hline 40 & Sitosterol alpha1 & 36.91 & 0.75 & Persicae Semen \\
\hline 41 & Gibberellin A44 & 101.61 & 0.54 & Persicae Semen \\
\hline 42 & 3-O-p-coumaroylquinic acid & 37.63 & 0.29 & Persicae Semen \\
\hline 43 & Folinic acid & 68.96 & 0.71 & Persicae Semen \\
\hline 44 & Linoleic acid & 41.90 & 0.14 & Pheretima \\
\hline 45 & Arachidonic acid & 45.57 & 0.20 & Pheretima \\
\hline 46 & Dihydrocapsaicin & 47.07 & 0.19 & Pheretima \\
\hline
\end{tabular}

OB, oral bioavailability; DL, drug-likeness. 


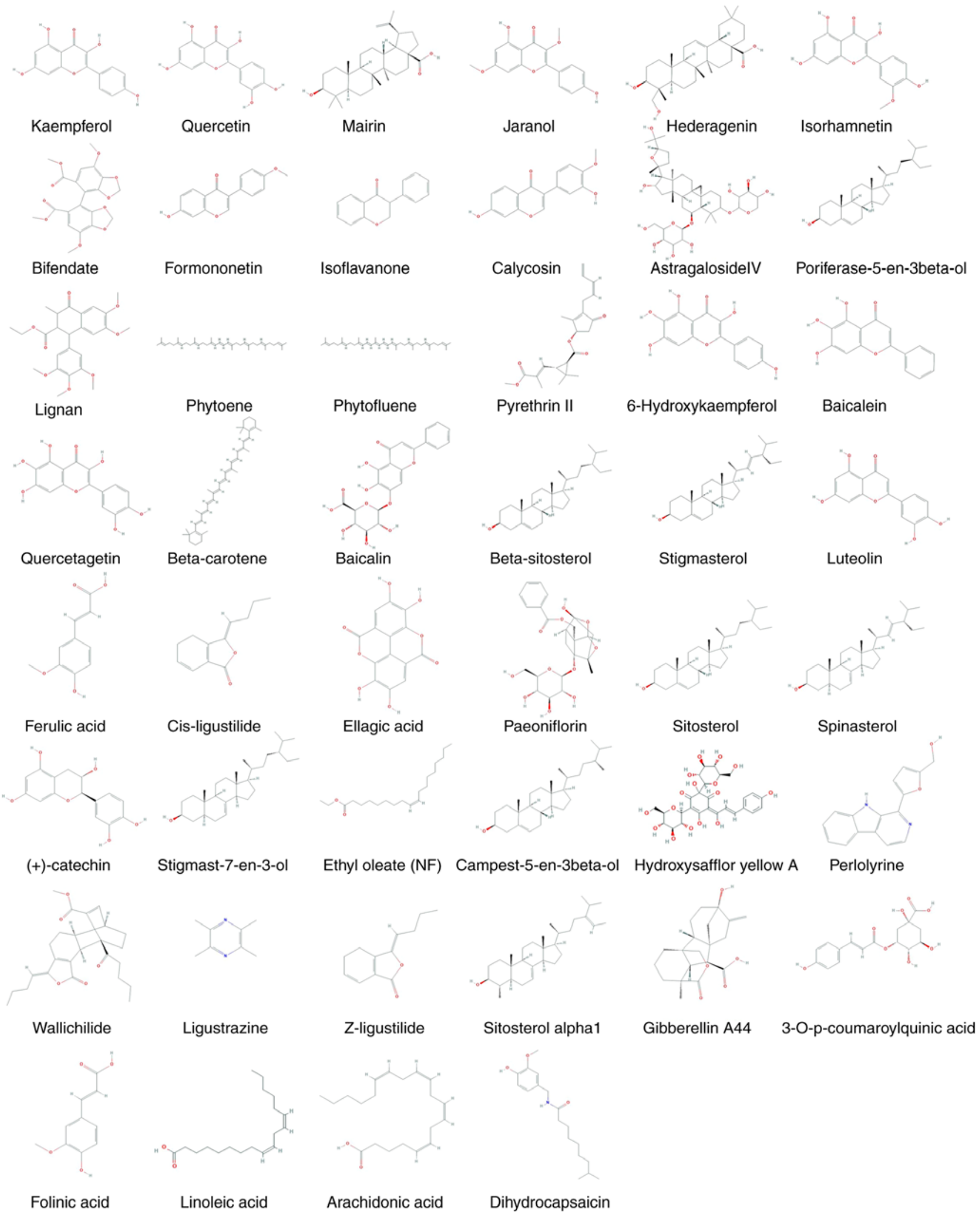

Figure 2. Final selected compound structures of Buyang Huanwu Decoction.

to prevent damage to the brain, except for tissue plasminogen activator; however, this single compound or single target drug has limited efficacy. Therefore, it is thought that a promising treatment approach for ischemic stroke should utilize multiple-component agents with multiple targets (27).
BYHWD activates blood circulation. It is a classic prescription that has been used by doctors for many generations. TCM cures disease using a multi-approach, multi-target and integer concept. It has unique advantages for the treatment of complex diseases, but due to the complex composition of TCM, its 


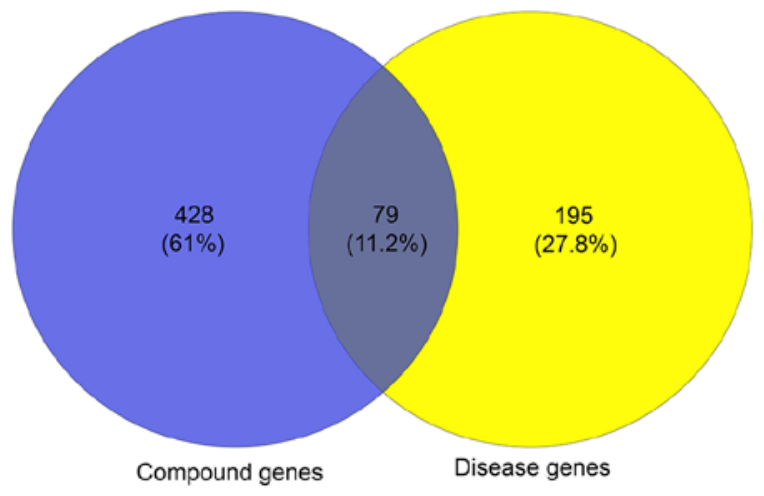

Figure 3. Venn diagram for compound genes and disease genes, 79 targets were common to Buyang Huanwu Decoction (compound genes) and cerebral ischemia (disease genes).

application in modern medicine has been slow. Furthermore, network pharmacology has provided a novel method of determining the pharmacological mechanisms of TCMs. Scholars have attempted to apply network pharmacology to evaluate the ingredients, targets and mechanisms of herbal formulas (8).

In the present study, network pharmacology was performed to investigate the pharmacological mechanisms of BYHWD in relation to cerebral ischemia. The network pharmacological analysis of BYHWD identified seven herbs, 42 compounds and 79 target gene-regulated pathways associated with cerebral ischemia.Additionally, 16compounds (baicalein, beta-carotene, baicalin, kaempferol, luteolin, quercetin, hydroxysafflor yellow A, isorhamnetin, bifendate, formononetin, calycosin, astragaloside IV, stigmasterol, sitosterol, Z-ligustilide and dihydrocapsaicin) were associated with $>5$ genes. Previous studies have reported that baicalein has potent neuroprotective properties under in vitro and in vivo systems (28). Beta-carotene serves as an antioxidant, inhibiting free radical production. It may also regulate cell growth and death (29). Baicalin inhibits microglial cell activation and reduces inflammation, oxidative damage and brain edema (30). In addition, kaempferol has strong anti-inflammatory and antioxidant effects. Numerous scientific reports have revealed that it serves a beneficial role in different inflammatory-related diseases, such as cardiovascular and neurodegenerative diseases. Luteolin suppresses inflammation in the brain tissue and regulates different cell signaling pathways (31). Quercetin has antioxidant stress and neuroprotective effects (32). Moreover, hydroxysafflor yellow A protects BMECs against OGD/reoxygenation-induced injury by inhibiting autophagy via the Class IPI3K/Akt/mTOR signaling pathway (33). Treatment of experimental stroke mice with isorhamnetin attenuated cerebral edema, improved blood-brain barrier function and upregulated the gene expression of certain tight junction proteins, including occludin, zonula occluden-1 and claudin-5 (34). Furthermore, calycosin protected the rat brain against ischemic injury by inhibiting calpain activation (35). Dihydrocapsaicin-treated cerebral ischemia-reperfusion rats demonstrated attenuated cerebral and blood-brain barrier damage by inhibiting oxidative stress and inflammatory pathways (36). These findings suggest that the main components of BYHWD are effective for treating cerebral ischemia. However, as the compounds in the database may be incomplete, the predicted active ingredients of
BYHWD may also be incomplete, which is a limitation of network pharmacology.

Genes with high degrees of differential articulation were acquired as a result of the PPI system analysis of the current study. Of these, IL6, TNF, VEGFA, HIF-1 $\alpha$, MAPK1, MAPK3, JUN, STAT3, IL1B and IL10 were recognized as the center genes. IL6 is a multifunctional cytokine with a wide range of biological activities, including regulation of the immune system and generation of acute phase reactions (37). VEGF is a pleiotropic growth factor that is crucially involved in neurovascular remodeling in the ischemic brain. VEGF promotes angiogenesis, protects ischemic neurons from injury, has potent anti-inflammatory actions and promotes brain plasticity (38). HIF-1 $\alpha$ regulates the expression of gene encoding molecules that participate in erythropoiesis, cell proliferation and energy metabolism, and is closely associated with the regulation of neuronal survival in ischemia $(39,40)$. HIF-1 $\alpha$ can upregulate the expression of proteins associated with the vascular system and can promote the angiogenesis of VEGF and its receptors to increase blood flow and reduce ischemic injury (41). TNF is a typical cytokine involved in the acute phase of systemic inflammation and is closely associated with the severity of cerebral ischemia (42). IL10 is a potent anti-inflammatory mediator and, if overexpressed, can suppress neuronal degeneration (43).

GO and KEGG pathway analyses were used to further understand the interaction and action pathways of target genes. GO analysis revealed that target genes were strongly associated with the following BPs: 'positive regulation of transcription including ribonucleic acid (RNA) polymerase II promoter', 'inflammatory response', 'transcription', 'DNA-templated', 'negative regulation of the apoptotic process', 'positive regulation of transcription', 'angiogenesis' and 'response to hypoxia'. The enriched MF ontologies were 'DNA binding transcription factor activity', 'sequence-specific DNA binding', 'identical protein binding', 'heparin-binding' and 'heme-binding'. The KEGG pathway analysis primarily pertained to TNF, IL17, apoptosis, PI3K-Akt, TLR, MAPK, NF- $\mathrm{B}$ and the HIF-1 signaling pathway. The results indicated that these pathways may interact to exert their combined effects against cerebral ischemia, which could explain the apparent effects of BYHWD.

Cerebral ischemia results in decreased cerebral blood flow and decreased oxygen supply, which leads to HIF-1 signaling pathway activation and upregulated HIF-1 $\alpha$ expression. This helps to recover blood circulation in the penumbra after cerebral ischemia, transport glucose and mediate hypoxia adaptation after hypoxia, serving a protective role in promoting cell survival and inhibiting brain tissue apoptosis (44). The MAPK and PI3K/Akt signaling pathways are the main pathways related to apoptosis after cerebral ischemia. Currently, it is hypothesized that MAPK signaling serves a dual role in the process of cell apoptosis, while PI3K/Akt signaling is important to the cell survival signaling pathway (45). Multiple neurotrophic factors inhibit apoptosis by activating the PI3K/Akt signaling pathway, thus playing a protective role in the brain (45). The PI3K/Akt signaling pathway is involved in the regulation of various intracellular signaling pathways and serves a key role in promoting cell survival and proliferation, anti-apoptosis, regulating glucose metabolism and protein synthesis (46). TLRs, as inflammatory signal receptors, 


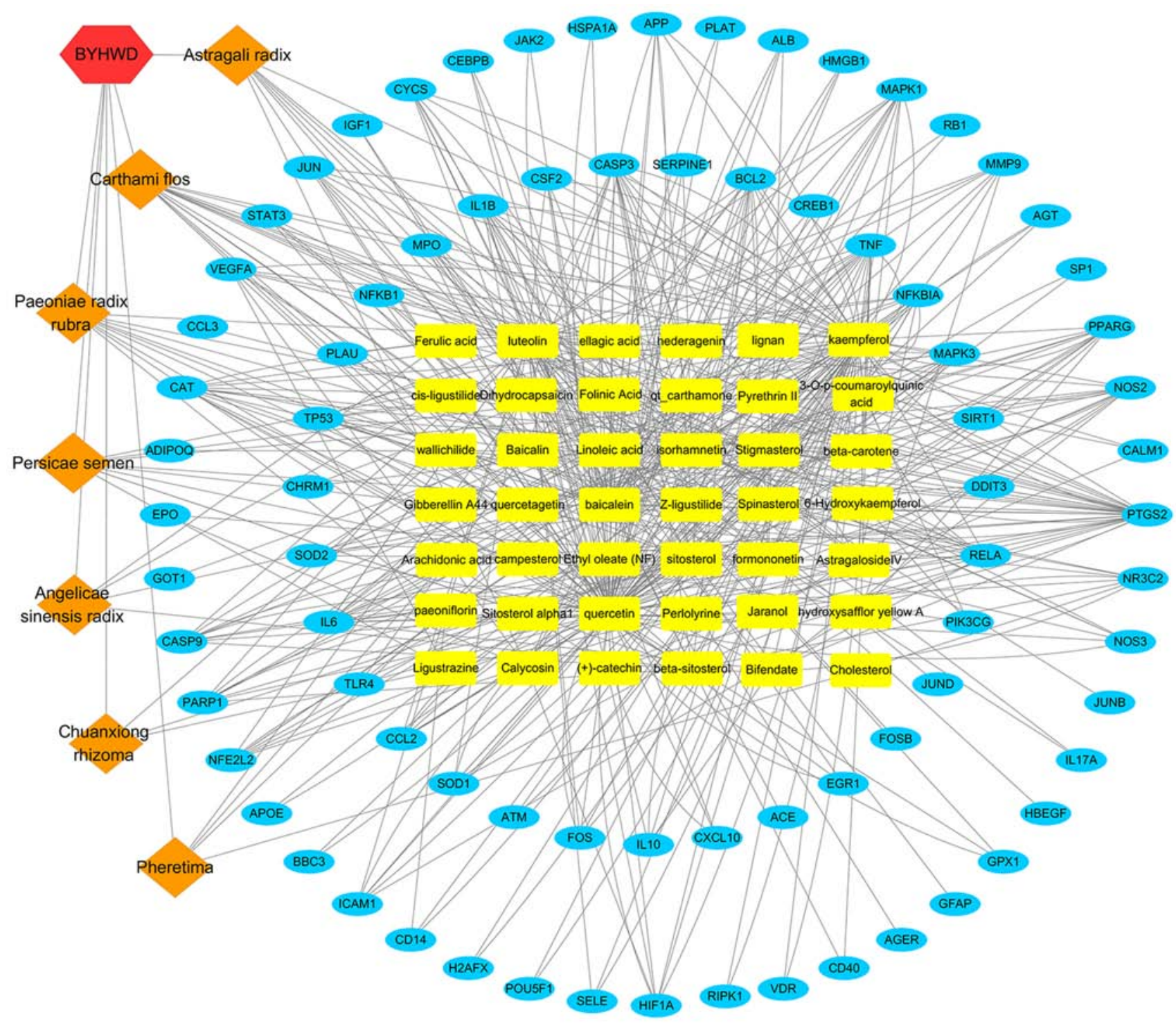

Figure 4. Network of BYHWD. Seven herbal medicines and 42 compounds were predicted to have 79 common potential protein targets. The common-target network included 129 nodes and 490 edges. Blue represents protein targets, orange represents the herbal medicines, yellow represents the compounds and red represents BYHWD. BYHWD, Buyang Huanwu Decoction.

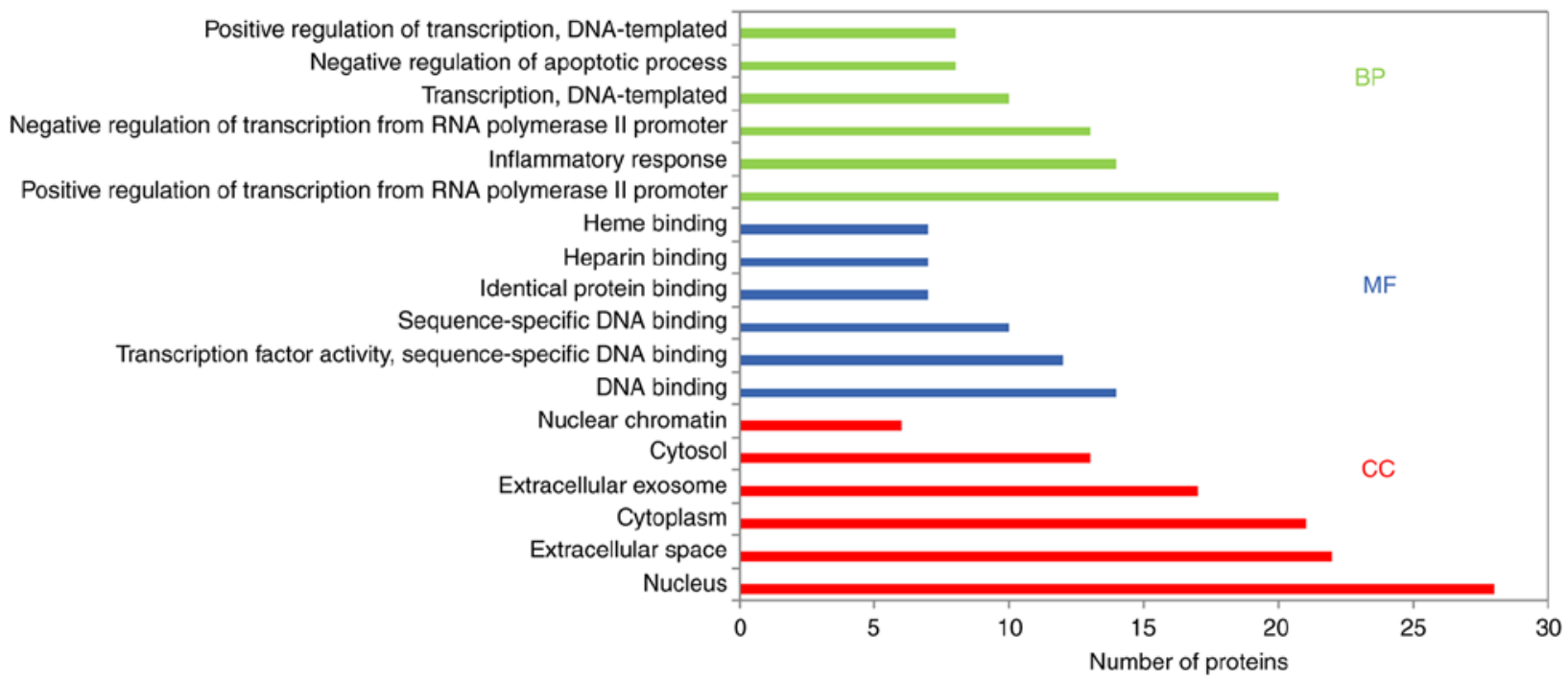

Figure 5. Gene Ontology analysis of candidate targets. The database revealed the top six enriched items in the BP, CC and MF categories. BP, biological process; CC, cell component; MF, molecular function. 
Table II. Biological processes of potential target genes based on GO enrichment analysis.

GO ID Biological process Genes P-value

GO:0045944 Positive regulation of transcription from RNA polymerase II promoter

GO:0006954 Inflammatory response

GO:0000122 Negative regulation of transcription from RNA polymerase II promoter

GO:0006351 Transcription, DNA-templated

GO:0043066 Negative regulation of apoptotic process

GO:0045893 Positive regulation of transcription, DNA-templated

GO:0031663 Lipopolysaccharide-mediated signaling pathway

GO:0001525 Angiogenesis

GO:0042127 Regulation of cell proliferation

GO:0006357 Regulation of transcription from RNA polymerase II promoter

GO:0001666 Response to hypoxia

GO:0051092 Positive regulation of NF-kappaB transcription factor activity

GO:0006915 Apoptotic process

GO:0055114 Oxidation-reduction process

GO:0006955 Immune response

GO:0043491 Protein kinase B signaling

GO:0071356 Cellular response to lipopolysaccharide

GO:0006979 Response to oxidative stress

GO:0045766 Positive regulation of angiogenesis

GO:0043524 Negative regulation of neuron apoptotic process
HMGB1, TNF, RELA, PPARG, TP53, IGF1, NFKBIA, $\quad 6.60 \times 10^{-12}$ RB1, CD40, SIRT1, IL10, VDR, IL17A, APP, HIF-1 $\alpha$, SP1, POU5F1, RIPK1, JUN and MAPK3

PIK3CG, HMGB1, CCL2, PTGS2, RELA, TLR4, CD40, AGER, IL10, CXCL10, IL17A, JAK2,

NFE2L2 and CD14

EGR1, VDR, TNF, POU5F1, RELA, JUN, PPARG, TP53, NFKB1, RB1, SIRT1, DDIT3 and EPO EGR1, VDR, HIF-1 $\alpha$, CEBPB, JUN, MAPK3, PPARG, TP53 and STAT3

CASP3, ALB, BCL2, TP53, IGF1, CAT, SIRT1 and STAT3

CEBPB, JUN, PPARG, NFKB1, NFE2L2, STAT3 and EPO

MAPK1, CCL2, TNF, MAPK3, NFKBIA, NOS3

and TLR4

PIK3CG, HIF-1 $\alpha$, JUN, VEGFA, SERPINE, NOS3 and SIRT1

JUN, NFKBIA, JAK2, NOS2, CD40, PLAU and CXCL10

FOS, SP1, NFKB1, RB1, NFE2L2, STAT3 and SOD2

PLAT, VEGFA, NOS2, AGER, PLAU and EPO

$7.35 \times 10^{-11}$

TNF, RELA, RIPK1, CAT, CD40 and AGER

$3.56 \times 10^{-7}$

$5.98 \times 10^{-5}$

$1.78 \times 10^{-4}$

$1.78 \times 10^{-4}$

$7.02 \times 10^{-10}$

$2.14 \times 10^{-5}$

$8.12 \times 10^{-5}$

$1.22 \times 10^{-3}$

$1.96 \times 10^{-5}$

$7.26 \times 10^{-5}$

APP, RIPK1, CYCS, TP53, RB1 and EPO

$2.98 \times 10^{-4}$

$1.43 \times 10^{-3}$

$6.95 \times 10^{-3}$

$1.21 \times 10^{-5}$

$1.82 \times 10^{-4}$

$2.91 \times 10^{-4}$

$6.75 \times 10^{-4}$

$7.56 \times 10^{-4}$

GO, Gene Ontology.

serve an important role in the inflammatory cascade reaction triggered by cerebral ischemia and are closely related to the expression of various inflammatory mediators (47). Therefore, it is of great significance to intervene in the TLR signaling pathway during the initial stage of the inflammatory response to effectively reduce inflammatory injury in the acute stage of ischemic stroke (47).

The current study verified three key targets (IL6, VEGFA and HIF-1 $\alpha$ ) using western blotting, which were predicted in the network. Therefore, the pharmacological mechanism of BYHWD in the treatment of ischemic stroke can be more clearly verified. The results revealed that when compared with the control group, BYHWD significantly inhibited the expression of IL6 and increased the expression of HIF-1 $\alpha$ and VEGFA.
In conclusion, the network pharmacology analysis of BYHWD identified seven herbs, 42 compounds and 79 target genes that were associated with ischemic stroke. The current study did not validate the identified active components, which was a limitation of the study. As the results of network pharmacology analysis revealed that baicalein, beta-carotene, baicalin, kaempferol, luteolin, quercetin, hydroxysafflor yellow A, isorhamnetin, bifendate, formononetin, calycosin, astragaloside IV, stigmasterol, sitosterol, Z-ligustilide and dihydrocapsaicin were the main effective components of BYHWD in the treatment of ischemic stroke, subsequent studies should first verify these results. Based on the pathway enrichment results of the present study, it was determined that the effects of BYHWD against ischemic stroke may be due to some of the ingredients that can simultaneously target multiple 
Table III. Potential target gene function based on Kyoto Encyclopedia of Genes and Genomes pathway analysis.

\begin{tabular}{|c|c|c|c|}
\hline KEGG ID & Pathway & Pathway genes & P-value \\
\hline hsa05167 & $\begin{array}{l}\text { Kaposi sarcoma-associated } \\
\text { herpesvirus infection }\end{array}$ & $\begin{array}{l}\text { PTGS2/CALM1/PIK3CG/RELA/RB1/MAPK1/MAPK3/ } \\
\text { NFKBIA/CASP3/CSF2/CYCS/JUN/NFKB1/STAT3/TP53/ } \\
\text { VEGFA/IL6/CASP9/ICAM1/FOS/HIF-1 } \alpha \text { /CREB1/JAK2 }\end{array}$ & $1.55 \times 10^{-19}$ \\
\hline hsa04668 & TNF signaling pathway & $\begin{array}{l}\text { PTGS2/RELA/MMP9/MAPK1/MAPK3/NFKBIA/TNF/ } \\
\text { CASP3/CEBPB/CSF2/IL1B/JUN/NFKB1/IL6/ICAM1/ } \\
\text { CCL2/FOS/SELE/RIPK1/CXCL10/JUNB/CREB1 }\end{array}$ & $2.80 \times 10^{-23}$ \\
\hline hsa05163 & $\begin{array}{l}\text { Human cytomegalovirus } \\
\text { infection }\end{array}$ & $\begin{array}{l}\text { PTGS2/CALM1/RELA/RB1/MAPK1/MAPK3/NFKBIA/ } \\
\text { TNF/CASP3/CYCS/IL1B/NFKB1/STAT3/TP53/VEGFA/ } \\
\text { IL6/CASP9/CCL2/RIPK1/SP1/CREB1/CCL3 }\end{array}$ & $1.83 \times 10^{-16}$ \\
\hline hsa04933 & $\begin{array}{l}\text { AGE-RAGE signaling pathway } \\
\text { in diabetic complications }\end{array}$ & $\begin{array}{l}\text { RELA/MAPK1/MAPK3/TNF/BCL2/CASP3/IL1B/JUN/ } \\
\text { NFKB1/STAT3/VEGFA/IL6/ICAM1/CCL2/SELE/ } \\
\text { AGER/EGR1/NOS3/AGT/SERPINE1/JAK2 }\end{array}$ & $7.02 \times 10^{-23}$ \\
\hline hsa04657 & IL17 signaling pathway & $\begin{array}{l}\text { PTGS2/RELA/MMP9/MAPK1/MAPK3/NFKBIA/TNF/ } \\
\text { CASP3/CEBPB/CSF2/IL1B/JUN/NFKB1/IL6/CCL2/FOS/ } \\
\text { CXCL10/FOSB/IL17A/JUND }\end{array}$ & $5.04 \times 10^{-22}$ \\
\hline hsa05161 & Hepatitis B & $\begin{array}{l}\text { RELA/MMP9/RB1/MAPK1/MAPK3/NFKBIA/TNF/BCL2/ } \\
\text { CASP3/CYCS/JUN/NFKB1/STAT3/TP53/IL6/TLR4/CASP9/ } \\
\text { FOS/CREB1/JAK2 }\end{array}$ & $6.08 \times 10^{-17}$ \\
\hline hsa05152 & Tuberculosis & $\begin{array}{l}\text { CALM1/NOS2/RELA/MAPK1/MAPK3/TNF/BCL2/CASP3/ } \\
\text { CEBPB/CYCS/IL1B/NFKB1/IL6/TLR4/CASP9/CD14/VDR/ } \\
\text { IL10/CREB1/JAK2 }\end{array}$ & $3.94 \times 10^{-16}$ \\
\hline hsa05145 & Toxoplasmosis & $\begin{array}{l}\text { NOS2/PIK3CG/RELA/MAPK1/MAPK3/NFKBIA/TNF/ } \\
\text { BCL2/CASP3/CYCS/NFKB1/STAT3/TLR4/CASP9/IL10/ } \\
\text { CD40/HSPA1A/JAK2 }\end{array}$ & $2.25 \times 10^{-17}$ \\
\hline hsa04210 & Apoptosis & $\begin{array}{l}\text { RELA/MAPK1/MAPK3/NFKBIA/TNF/BCL2/CASP3/ } \\
\text { CYCS/JUN/NFKB1/TP53/CASP9/PARP1/BBC3/ATM/FOS/ } \\
\text { RIPK1/DDIT3 }\end{array}$ & $6.85 \times 10^{-16}$ \\
\hline hsa05142 & $\begin{array}{l}\text { Chagas disease (American } \\
\text { trypanosomiasis) }\end{array}$ & $\begin{array}{l}\text { NOS2/RELA/MAPK1/MAPK3/NFKBIA/TNF/IL1B/JUN/ } \\
\text { NFKB1/IL6/TLR4/CCL2/FOS/IL10/ACE/ SERPINE1/CCL3 }\end{array}$ & $1.03 \times 10^{-16}$ \\
\hline hsa05418 & $\begin{array}{l}\text { Fluid shear stress and } \\
\text { atherosclerosis }\end{array}$ & $\begin{array}{l}\text { CALM1/RELA/MMP9/TNF/BCL2/IL1B/JUN/NFKB1/TP53/ } \\
\text { VEGFA/NFE2L2/ICAM1/CCL2/FOS/SELE/ NOS3/PLAT }\end{array}$ & $1.87 \times 10^{-14}$ \\
\hline hsa05202 & $\begin{array}{l}\text { Transcriptional misregulation } \\
\text { in cancer }\end{array}$ & $\begin{array}{l}\text { PPARG/RELA/MMP9/CEBPB/CSF2/IGF1/MPO/NFKB1/ } \\
\text { PLAU/TP53/IL6/ATM/CD14/CD40/SP1/DDIT3/PLAT }\end{array}$ & $2.41 \times 10^{-12}$ \\
\hline hsa05169 & Epstein-Barr virus infection & $\begin{array}{l}\text { RELA/RB1/NFKBIA/TNF/BCL2/CASP3/CYCS/JUN/ } \\
\text { NFKB1/STAT3/TP53/IL6/CASP9/ICAM1/RIPK1/CD40/ } \\
\text { CXCL10 }\end{array}$ & $8.51 \times 10^{-12}$ \\
\hline hsa05166 & $\begin{array}{l}\text { Human T-cell leukemia virus } 1 \\
\text { infection }\end{array}$ & $\begin{array}{l}\text { RELA/RB1/MAPK1/MAPK3/NFKBIA/TNF/CSF2/JUN/ } \\
\text { NFKB1/TP53/IL6/ICAM1/ATM/FOS/CD40/EGR1/CREB1 }\end{array}$ & $3.38 \times 10^{-11}$ \\
\hline hsa04151 & PI3K-Akt signaling pathway & $\begin{array}{l}\text { PIK3CG/RELA/MAPK1/MAPK3/BCL2/IGF1/NFKB1/TP53/ } \\
\text { VEGFA/IL6/TLR4/CASP9/NOS3/CREB1/JAK2/EPO/ } \\
\text { CHRM1 }\end{array}$ & $5.39 \times 10^{-8}$ \\
\hline hsa04620 & $\begin{array}{l}\text { Toll-like receptor signaling } \\
\text { pathway }\end{array}$ & $\begin{array}{l}\text { RELA/MAPK1/MAPK3/NFKBIA/TNF/IL1B/JUN/NFKB1/ } \\
\text { IL6/TLR4/CD14/FOS/RIPK1/CD40/CXCL10/CCL3 }\end{array}$ & $2.89 \times 10^{-15}$ \\
\hline hsa05162 & Measles & $\begin{array}{l}\text { RELA/NFKBIA/BCL2/CASP3/CYCS/IL1B/JUN/NFKB1/ } \\
\text { STAT3/TP53/IL6/TLR4/CASP9/BBC3/FOS/HSPA1A }\end{array}$ & $2.81 \times 10^{-13}$ \\
\hline hsa05164 & Influenza A & $\begin{array}{l}\text { RELA/MAPK1/MAPK3/NFKBIA/TNF/CASP3/CYCS/IL1B/ } \\
\text { NFKB1/IL6/TLR4/CASP9/ICAM1/CCL2/CXCL10/JAK2 }\end{array}$ & $5.60 \times 10^{-12}$ \\
\hline hsa05170 & $\begin{array}{l}\text { Human immunodeficiency } \\
\text { virus } 1 \text { infection }\end{array}$ & $\begin{array}{l}\text { CALM1/RELA/MAPK1/MAPK3/NFKBIA/TNF/BCL2/ } \\
\text { CASP3/CYCS/JUN/NFKB1/TLR4/CASP9/ATM/FOS/RIPK1 }\end{array}$ & $2.12 \times 10^{-10}$ \\
\hline hsa04010 & MAPK signaling pathway & $\begin{array}{l}\text { RELA/MAPK1/MAPK3/TNF/CASP3/IGF1/IL1B/JUN/ } \\
\text { NFKB1/TP53/VEGFA/CD14/FOS/JUND/DDIT3/HSPA1A }\end{array}$ & $2.60 \times 10^{-8}$ \\
\hline hsa05133 & Pertussis & $\begin{array}{l}\text { CALM1/NOS2/RELA/MAPK1/MAPK3/TNF/CASP3/IL1B/ } \\
\text { JUN/NFKB1/IL6/TLR4/CD14/FOS/IL10 }\end{array}$ & $1.55 \times 10^{-1}$ \\
\hline
\end{tabular}


Table III. Continued.

\begin{tabular}{llll}
\hline KEGG ID & \multicolumn{1}{c}{ Pathway } & Pathway genes & P-value \\
\hline hsa04064 & NF-kappa B signaling pathway & PTGS2/RELA/NFKBIA/TNF/BCL2/IL1B/NFKB1/PLAU/ & $2.80 \times 10^{-23}$ \\
& & TLR4/PARP1/ICAM1/ATM/CD14/RIPK1/CD40 & \\
hsa04066 & HIF-1 signaling pathway & NOS2/RELA/MAPK1/MAPK3/BCL2/IGF1/NFKB1/STAT3/ & $1.83 \times 10^{-16}$ \\
& & VEGFA/IL6/TLR4/HIF-1 $/$ /NOS3/SERPINE1/EPO & \\
\hline
\end{tabular}

A

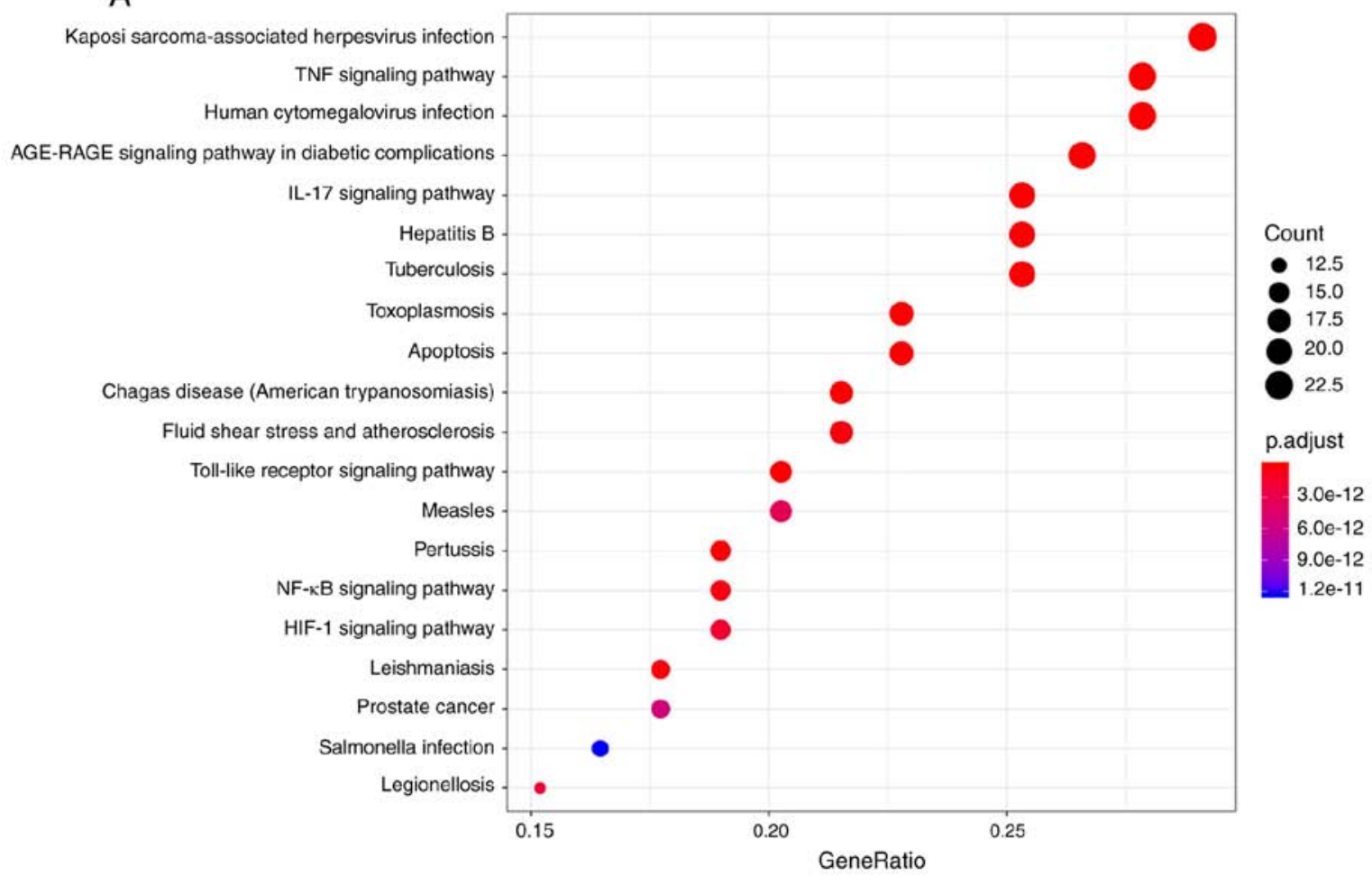

B

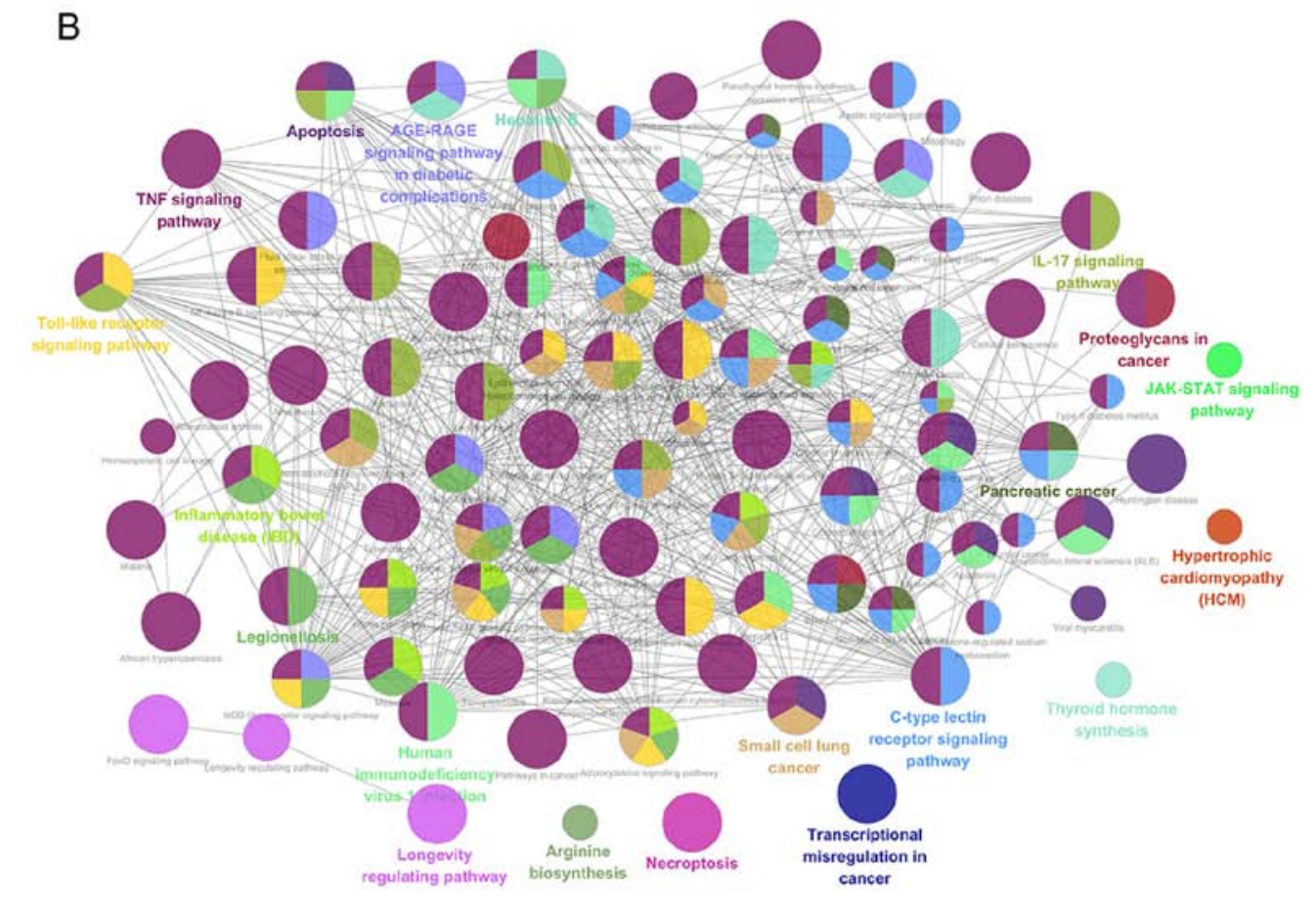

Figure 6. Buyang Huanwu Decoction in the treatment of cerebral ischemia signaling pathway. (A) KEGG pathways of target genes and (B) main functional annotation clusters determined through Biocarta analysis. 

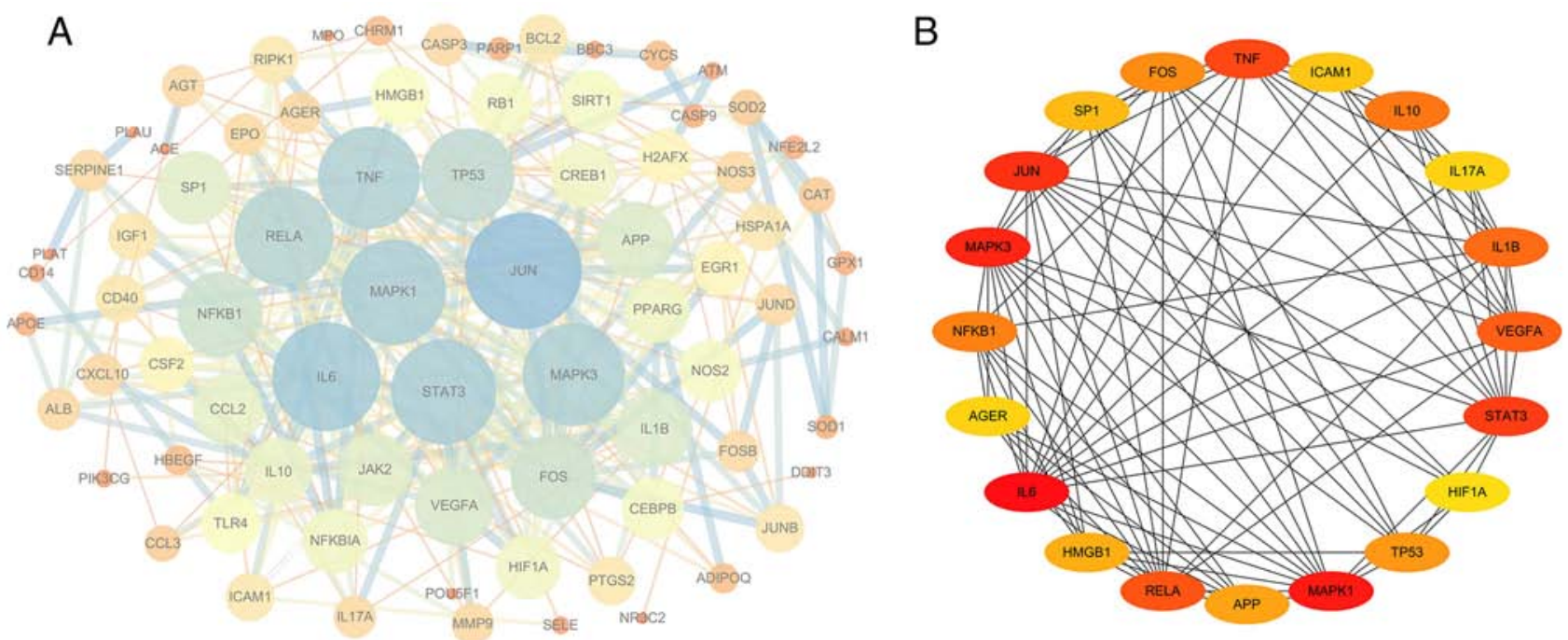

Figure 7. PPI networks of the active ingredients of Buyang Huanwu Decoction for the treatment of cerebral ischemia. (A) The network of PPI relationships had 79 nodes and 327 edges, with an average node degree of 8.28. Each node represented the relevant gene. The edge and line thickness indicated the strength of supporting data. (B) The top 20 hub genes in the PPI network are presented. The darker the color, the higher the score. PPI, protein-protein interaction.
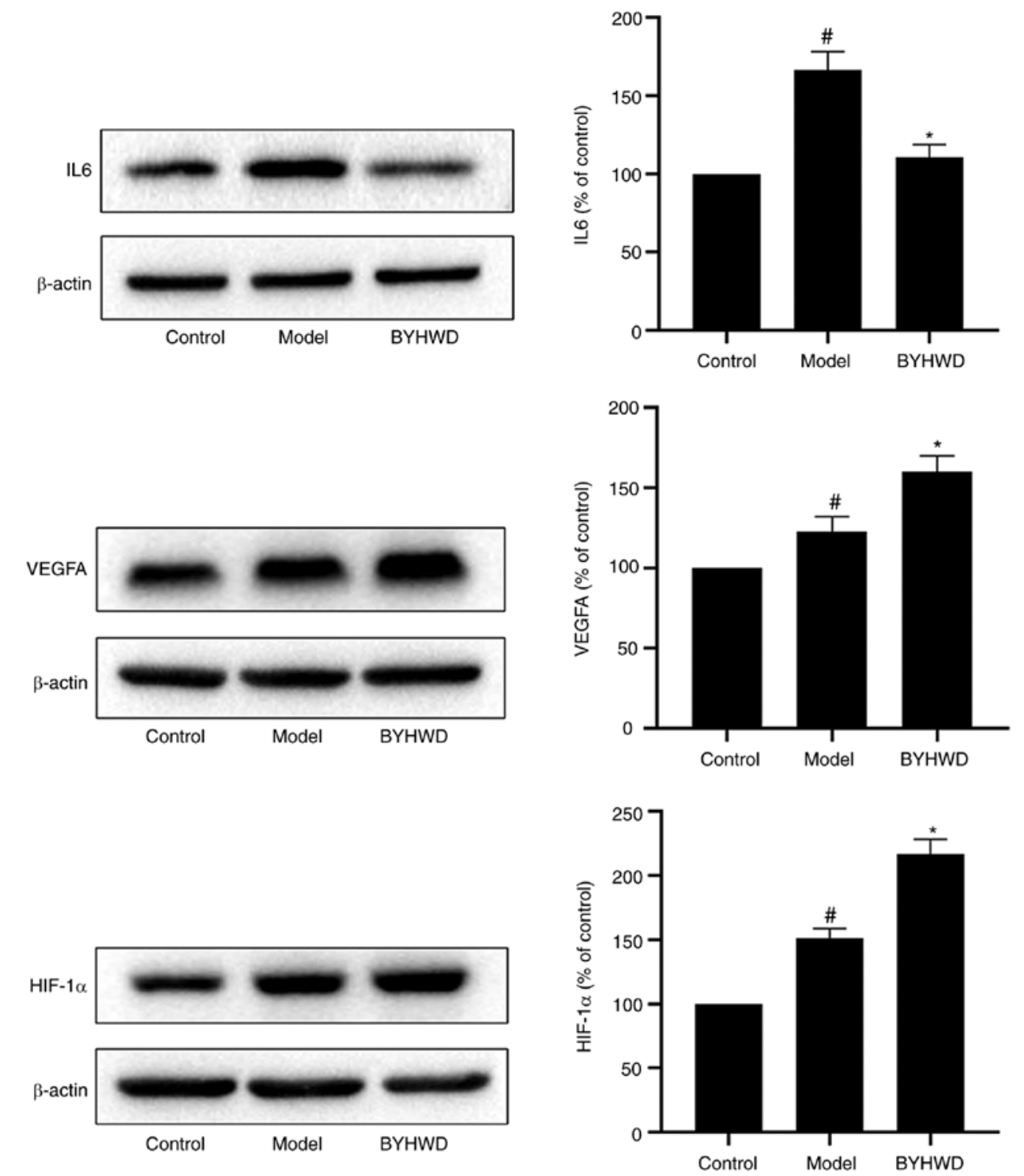

Figure 8. Effects of BYHWD on the levels of IL6, VEGFA and HIF-1 $\alpha$ in brain microvascular endothelial cells. Data were expressed as the mean \pm SD. ${ }^{*} \mathrm{P}<0.05$ vs. the control group. "P<0.05 vs. the model group. BYHWD, Buyang Huanwu Decoction; HIF-1 $\alpha$, hypoxia-inducible-factor- $1 \alpha$. 
pathways, such as the TNF, IL17, apoptosis, PI3K-Akt, TLR, MAPK, NF- $\kappa$ B and HIF-1 signaling pathways. Furthermore, genes with high degrees of differential articulation were identified from PPI system analysis. IL6, TNF, VEGFA, HIF-1 $\alpha$, MAPK1, MAPK3, JUN, STAT3, IL1B and IL10 were recognized as center genes. The results indicated that compound-target gene networks can reveal close interactions between multi-components and multi-targets, enhancing understanding of the potential effects of BYHWD in ischemic stroke.

\section{Acknowledgements}

Not applicable.

\section{Funding}

This research was funded by the National Natural Science Foundation of China (grant nos. 81503280 and 81573549).

\section{Availability of data and materials}

The datasets used and/or analyzed during the current study are available from the corresponding author on reasonable request.

\section{Authors' contributions}

KW, LL, JC and YQ designed the experiments. KW and LL conducted the experiments and researched the literature. $\mathrm{KW}$, JC, YQ and RL collected and analyzed the data. KW wrote the manuscript. KW, JC and ZY revised the manuscript. JD, ZF, YD and YM interpreted the data. ZY and EZ obtained funding and designed the study. KW and LL confirm the authenticity of all the raw data. All authors read and approved the final manuscript.

\section{Ethics approval and consent to participate}

Not applicable.

\section{Patient consent for publication}

Not applicable.

\section{Competing interests}

The authors declare that they have no competing interests.

\section{References}

1. Del Sette M, Chiti A and Dinia L: Intraarterial treatment for acute ischemic stroke. N Engl J Med 372: 1177, 2015.

2. Wang P, Shao BZ, Deng Z, Chen S, Yue Z and Miao CY: Autophagy in ischemic stroke. Prog Neurobiol 163-164: 98-117, 2018.

3. Writing Group Members, Mozaffarian D, Benjamin EJ, Go AS, Arnett DK, Blaha MJ, Cushman M, Das SR, de Ferranti S, Després JP, et al: Heart disease and stroke statistics-2016 update: A report From the American Heart Association. Circulation 133: e38-e360, 2016.

4. Hung IL, Hung YC, Wang LY, Hsu SF, Chen HJ, Tseng YJ, Kuo CE, Hu WL and Li TC: Chinese herbal products for ischemic stroke. Am J Chin Med 43: 1365-1379, 2015.
5. Li JH, Liu AJ, Li HQ, Wang Y, Shang HC and Zheng GQ: Buyang huanwu decoction for healthcare: Evidence-based theoretical interpretations of treating different diseases with the same method and target of vascularity. Evid Based Complement Alternat Med 2014: 506783, 2014.

6. Huang J, Guo W, Cheung F, Tan HY, Wang N and Feng Y: Integrating network pharmacology and experimental models to investigate the efficacy of Coptidis and Scutellaria containing Huanglian Jiedu decoction on hepatocellular carcinoma. Am J Chin Med 48: 161-182, 2020.

7. Zhang J, Liu X, Wu J, Zhou W, Tian J, Guo S, Jia SS, Meng Z and Ni M: A bioinformatics investigation into the pharmacological mechanisms of the effect of the Yinchenhao decoction on hepatitis $\mathrm{C}$ based on network pharmacology. BMC Complement Med Ther 20: 50, 2020.

8. Lee AY, Park W, Kang TW, Cha MH and Chun JM: Network pharmacology-based prediction of active compounds and molecular targets in Yijin-tang acting on hyperlipidaemia and atherosclerosis. J Ethnopharmacol 221: 151-159, 2018.

9. Li XK, Yang HJ, Xiao JC, Zhang J, Zhang J, Liu M, Zheng Y and Ma L: Network pharmacology based investigation into the bioactive compounds and molecular mechanisms of Schisandrae chinensis Fructus against drug-induced liver injury. Bioorg Chem 96: 103553, 2020.

10. Xu T, Li S, Sun Y, Pi Z, Liu S, Song F and Liu Z: Systematically characterize the absorbed effective substances of Wutou Decoction and their metabolic pathways in rat plasma using UHPLC-Q-TOF-MS combined with a target network pharmacological analysis. J Pharm Biomed Anal 141: 95-107, 2017.

11. Kim SK, Lee S, Lee MK and Lee S: A systems pharmacology approach to investigate the mechanism of Oryeong-san formula for the treatment of hypertension. J Ethnopharmacol 244: 112129, 2019.

12. Wang W, Liu T, Yang L, Ma Y, Dou F, Shi L, Wen A and Ding Y: Study on the multi-targets mechanism of Triphala on cardio-cerebral vascular diseases based on network pharmacology. Biomed Pharmacother 116: 108994, 2019.

13. Zhang J, Liang R, Wang L and Yang B: Effects and mechanisms of Danshen-Shanzha herb-pair for atherosclerosis treatment using network pharmacology and experimental pharmacology. J Ethnopharmacol 229: 104-114, 2019.

14. Xu T, Ma C, Fan S, Deng N, Lian Y, Tan L, Du W, Zhang S, Liu S, Ren B, et al: Systematic understanding of the mechanism of baicalin against Ischemic stroke through a network pharmacology approach. Evid Based Complement Alternat Med 2018: 2582843, 2018.

15. Piñero J, Bravo Â, Queralt-Rosinach N, Gutiérrez-Sacristán A, Deu-Pons J, Centeno E, García-García J, Sanz F and Furlong LI: DisGeNET: A comprehensive platform integrating information on human disease-associated genes and variants. Nucleic Acids Res 45: D833-D839, 2017.

16. Zhang J, Yang B, Wang L and Liang R: Effects and mechanisms of Danshen-Shanzha herb-pair for atherosclerosis treatment using network pharmacology and experimental pharmacology. J Ethnopharmacol 229: 104-114, 2019.

17. Rouillard A, Gundersen G, Fernandez N, Wang Z, Monteiro CD, McDermott MG and Ma'ayan A: The harmonizome: A collection of processed datasets gathered to serve and mine knowledge about genes and proteins. Database (Oxford) 2016: baw100, 2016.

18. Franceschini A, Szklarczyk D, Frankild S, Kuhn M, Simonovic M, Roth A, Lin J, Minguez P, Bork P, von Mering C and Jensen LJ: STRING v9.1: Protein-protein interaction networks, with increased coverage and integration. Nucleic Acids Res 41 (Database issue): D808-D815, 2013.

19. Qiao $\mathrm{P}$, Yan $\mathrm{H}$ and Wang J: EGb761 protects brain microvascular Endothelial cells against oxygen-glucose deprivation-induced injury through $\operatorname{lncRNA} \mathrm{Rmst} / \mathrm{miR}-150$ axis. Neurochem Res 45 : 2398-2408, 2020.

20. Sun Y, Xu DP, Qin Z, Wang PY, Hu BH, Yu JG, Zhao Y, Cai B, Chen YL, Lu M, et al: Protective cerebrovascular effects of hydroxysafflor yellow A (HSYA) on ischemic stroke. Eur J Pharmacol 818: 604-609, 2018.

21. Yin F, Zhou H, Fang Y, Li C, He Y, Yu L, Wan H and Yang J: Astragaloside IV alleviates ischemia reperfusion-induced apoptosis by inhibiting the activation of key factors in death receptor pathway and mitochondrial pathway. J Ethnopharmacol 248: $112319,2020$.

22. Fritsche KL: Linoleic acid, vegetable oils \& inflammation. Mo Med 111: 41-43, 2014.

23. Zdunska K, Dana A, Kolodziejczak A and Rotsztejn H: Antioxidant properties of ferulic acid and its possible application. Skin Pharmacol Physiol 31: 332-336, 2018. 
24. Li J, Yu J, Ma H, Yang N, Li L, Zheng DD, Wu MX, Zhao ZL and Qi HY: Intranasal pretreatment with Z-Ligustilide, the main volatile component of Rhizoma Chuanxiong, confers prophylaxis against cerebral ischemia via Nrf2 and HSP70 signaling pathways. J Agric Food Chem 65: 1533-1542, 2017.

25. Wei Y, Liu J, Zhang H, Du X, Luo Q, Sun J, Liu F, Li M, Xu F, Wei K and Dong J: Ligustrazine attenuates inflammation and the associated chemokines and receptors in ovalbumine-induced mouse asthma model. Environ Toxicol Pharmacol 46: 55-61, 2016.

26. Pandya RS, Mao L, Zhou H, Zhou S, Zeng J, Popp AJ and Wang X: Central nervous system agents for ischemic stroke: Neuroprotection mechanisms. Cent Nerv Syst Agents Med Chem 11: 81-97, 2011.

27. Zhang WW, Xu F, Wang D, Ye J and Cai SQ: Buyang Huanwu Decoction ameliorates ischemic stroke by modulating multiple targets with multiple components: In vitro evidences. Chin J Nat Med 16: 194-202, 2018.

28. Sowndhararajan K, Deepa P, Kim M, Park SJ and Kim S Baicalein as a potent neuroprotective agent: A review. Biomed Pharmacother 95: 1021-1032, 2017.

29. Palozza P: Can beta-carotene regulate cell growth by a redox mechanism? An answer from cultured cells. Biochim Biophys Acta 1740: 215-221, 2005

30. Shi X, Fu Y, Zhang S, Ding H and Chen J: Baicalin attenuates subarachnoid hemorrhagic brain injury by modulating blood-brain barrier disruption, Inflammation, and oxidative damage in mice. Oxid Med Cell Longev 2017: 1401790 2017.

31. Nabavi SF, Braidy N, Gortzi O, Sobarzo-Sanchez E, Daglia M, Skalicka-Woźniak K and Nabavi SM: Luteolin as an antiinflammatory and neuroprotective agent: A brief review. Brain Res Bull 119: 1-11, 2015.

32. Barreca D, Bellocco E, D'Onofrio G, Nabavi SF, Daglia M, Rastrelli L and Nabavi SM: Neuroprotective effects of quercetin: From chemistry to medicine. CNS Neurol Disord Drug Targets 15: 964-975, 2016.

33. Yang G, Wang N, Seto SW, Chang D and Liang H: Hydroxysafflor yellow a protects brain microvascular endothelial cells against oxygen glucose deprivation/reoxygenation injury: Involvement of inhibiting autophagy via class I PI3K/Akt/mTOR signaling pathway. Brain Res Bull 140: 243-257, 2018.

34. Zhao JJ, Song JQ, Pan SY and Wang K: Treatment with isorhamnetin protects the brain against ischemic injury in mice. Neurochem Res 41: 1939-1948, 2016.

35. Guo C, Ma Y, Ma S, Mu F, Deng J, Duan J, Xiong L, Yin Y, Wang Y, $\mathrm{Xi} \mathrm{M}$ and Wen A: The role of TRPC6 in the neuroprotection of calycosin against cerebral ischemic injury. Sci Rep 7: 3039, 2017.
36. Janyou A, Wicha P, Jittiwat J, Suksamrarn A, Tocharus C and Tocharus J: Dihydrocapsaicin attenuates blood brain barrier and cerebral damage in focal cerebral ischemia/reperfusion via oxidative stress and inflammatory. Sci Rep 7: 10556, 2017.

37. Robson-Ansley P, Cockburn E, Walshe I, Stevenson E and Nimmo M: The effect of exercise on plasma soluble IL-6 receptor concentration: A dichotomous response. Exerc Immunol Rev 16: 56-76, 2010.

38. Ma Y,Zechariah A, Qu Y and Hermann DM: Effects of vascular endothelial growth factor in ischemic stroke. J Neurosci Res 90: 1873-1882, 2012.

39. Ciurea AV, Palade C, Voinescu D and Nica DA: Subarachnoid hemorrhage and cerebral vasospasm - literature review. J Med Life 6: 120-125, 2013.

40. Guo Y: Role of HIF-1a in regulating autophagic cell survival during cerebral ischemia reperfusion in rats. Oncotarget 8: 98482-98494, 2017.

41. Chen ZZ, Gong X, Guo Q, Zhao H and Wang L: Bu Yang Huan $\mathrm{Wu}$ Decoction prevents reperfusion injury following ischemic stroke in rats via inhibition of HIF-1 $\alpha$, VEGF and promotion $\beta$-ENaC expression. J Ethnopharmacol 228: 70-81, 2019.

42. Oliveira DMG, Aguiar LT, de Oliveira Limones MV, Gomes AG, da Silva LC, de Morais Faria CDC and Scalzo PL: Aerobic training efficacy in inflammation, neurotrophins, and function in chronic stroke persons: A randomized controlled trial protocol. J Stroke Cerebrovasc Dis 28: 418-424, 2019.

43. Nakajima M, Nito C, Sowa K, Suda S, Nishiyama Y, Nakamura-Takahashi A, Nitahara-Kasahara Y, Imagawa K, Hirato T, Ueda M, et al: Mesenchymal stem cells overexpressing interleukin-10 promote neuroprotection in experimental acute ischemic stroke. Mol Ther Methods Clin Dev 6: 102-111, 2017.

44. Singh N, Sharma G and Mishra V: Hypoxia inducible factor-1: Its potential role in cerebral ischemia. Cell Mol Neurobiol 32: 491-507, 2012.

45. Franke TF: PI3K/Akt: Getting it right matters. Oncogene 27: 6473-6488, 2008.

46. Gao X, Zhang H, Takahashi T, Hsieh J, Liao J, Steinberg GK and Zhao H: The Akt signaling pathway contributes to postconditioning's protection against stroke; the protection is associated with the MAPK and PKC pathways. J Neurochem 105: 943-955, 2008.

47. Eltzschig HK and Eckle T: Ischemia and reperfusion-from mechanism to translation. Nat Med 17: 1391-1401, 2011.

This work is licensed under a Creative Commons Attribution-NonCommercial-NoDerivatives 4.0 International (CC BY-NC-ND 4.0) License. 